\title{
Cognitive Impairment in Cocaine Users is Drug-Induced but Partially Reversible: Evidence from a Longitudinal Study
}

\author{
Matthias Vonmoos*,', Lea M Hulka', Katrin H Preller', Franziska Minder', Markus R Baumgartner ${ }^{2}$ and \\ Boris B Quednow*,I \\ 'Experimental and Clinical Pharmacopsychology, Department of Psychiatry, Psychotherapy, and Psychosomatics, Psychiatric Hospital, University \\ of Zurich, Zurich, Switzerland; ${ }^{2}$ Center of Forensic Hairanalytics, Institute of Forensic Medicine, University of Zurich, Zurich, Switzerland
}

\begin{abstract}
Cocaine users consistently display cognitive impairments. However, it is still unknown whether these impairments are cocaine-induced and if they are reversible. Therefore, we examined the relation between changing intensity of cocaine use and the development of cognitive functioning within I year. The present data were collected as part of the longitudinal Zurich Cocaine Cognition Study $\left(\mathrm{ZuCo}{ }^{2} \mathrm{St}\right)$. Forty-eight psychostimulant-naive controls and 57 cocaine users (19 with increased, 19 with decreased, and 19 with unchanged cocaine use) were eligible for analysis. At baseline and after a I-year follow-up, cognitive performance was measured by a global cognitive index and four neuropsychological domains (attention, working memory, declarative memory, and executive functions), calculated from 13 parameters of a broad neuropsychological test battery. Intensity of cocaine use was objectively determined by quantitative 6-month hair toxicology at both test sessions. Substantially increased cocaine use within I year (mean + 297\%) was associated with reduced cognitive performance primarily in working memory. By contrast, decreased cocaine use ( $-72 \%)$ was linked to small cognitive improvements in all four domains. Importantly, users who ceased taking cocaine seemed to recover completely, attaining a cognitive performance level similar to that of the control group. However, recovery of working memory was correlated with age of onset of cocaine use - early-onset users showed hampered recovery. These longitudinal data suggest that cognitive impairment might be partially cocaine-induced but also reversible within I year, at least after moderate exposure. The reversibility indicates that neuroplastic adaptations underlie cognitive changes in cocaine users, which are potentially modifiable in psychotherapeutical or pharmacological interventions.

Neuropsychopharmacology (20I4) 39, 2200-22I0; doi:I0.I038/npp.2014.7I; published online 23 April 20 I4
\end{abstract}

\section{INTRODUCTION}

The annual number of cocaine users is currently estimated at 17 million people worldwide (United Nations Office on Drugs and Crime, 2013). Because of its high addictive potential and harmful effects on mental and physical well-being (Degenhardt and Hall, 2012; Nutt et al, 2007), the use of cocaine is a major public health issue with substantial societal and economic costs (Degenhardt and Hall, 2012).

Accumulating evidence suggests that dependent (Goldstein et al, 2004; Jovanovski et al, 2005; Vonmoos et al, 2013;

\footnotetext{
* Correspondence: Dr M Vonmoos, Experimental and Clinical Pharmacopsychology, Department of Psychiatry, Psychotherapy, and Psychosomatics, Psychiatric Hospital, University of Zurich, Lenggstrasse 31, Zurich CH-8032, Switzerland, Tel: +4I 44384 2666, Fax: +4I 44384 3396, E-mail: matthias.vonmoos@bli.uzh.ch or DrBB Quednow, Experimental and Clinical Pharmacopsychology, Department of Psychiatry, Psychotherapy, and Psychosomatics, Psychiatric Hospital, University of Zurich, Lenggstrasse 31, Zurich CH-8032, Switzerland, Tel: +4I 443842777 , Fax: +4I 44384 3396, E-mail: quednow@bli.uzh.ch Received 18 September 2013; revised I4 March 2014; accepted 17 March 2014; accepted article preview online 2I March 2014
}

Woicik et al, 2009) and also recreational (Colzato et al, 2009; Reske et al, 2010; Soar et al, 2012; Vonmoos et al, 2013) cocaine use is associated with broad neuropsychological impairment. Remarkably, a first study indicates that $30 \%$ of dependent users and even $12 \%$ of recreational users exhibit clinically relevant global cognitive impairment (Vonmoos et al, 2013). Studies have shown deficits in attention, working memory, and declarative memory in chronic cocaine users, whereas the heterogeneous concept of executive functions has yielded mixed results (Jovanovski et al, 2005; Vonmoos et al, 2013). We recently demonstrated that cocaine users additionally display inferior social cognition, including prosodic and cross-modal emotion recognition, emotional empathy, mental perspective-taking, and social decision-making (Hulka et al, 2014, 2013; Preller et al, 2014). A worse social cognitive performance was correlated with a smaller social network and more criminal offenses in cocaine users (Preller et al, 2014), pointing to the importance of cognitive health for social and occupational functioning in drug users as in psychiatric patients (Lee et al, 2013). Moreover, neuropsychological performance predicts the attainment of treatment objectives and the likelihood of treatment dropout in substance users (Teichner et al, 2002). 
Today, it is still unclear whether these cognitive impairments are cocaine-induced and if they are reversible. Studies on chronic cocaine self-administration in rhesus monkeys suggest that some alterations in attention, learning, and working memory might be cocaine-induced (Gould et al, 2012; Liu et al, 2008; Porter et al, 2011). In contrast to these animal studies, research with human cocaine users has focused on the effects of drug abstinence on cognition. The few and preliminary cross-sectional (Bolla et al, 1999; De Oliveira et al, 2009) and longitudinal (Bauer, 1996; Bolla et al, 2000; Di Sclafani et al, 2002; van Gorp et al, 1999) studies either indicate persisting neuropsychological impairment in attention (Bauer, 1996), declarative memory (van Gorp et al, 1999), and executive function (De Oliveira et al, 2009) or suggest some recovery effects in working memory (Di Sclafani et al, 2002) and verbal declarative memory (De Oliveira et al, 2009). However, it should be noted that even longitudinal studies in humans cannot prove causal relationships between drug intake and cognition. Furthermore, cocaine use was self-reported and solely controlled with drug urine tests but not hair toxicology analyses, which would have enabled a reliable detection of drug use during the last months. Finally, these studies had relatively brief follow-up intervals with strongly varying abstinence durations ( 1 week to 6 months) and several studies reported only minimal information on the severity of drug use. Notably, no longitudinal study has investigated the association between escalating cocaine use and cognitive impairment yet.

Accordingly, we aimed to overcome these limitations of previous studies by means of a longitudinal study specifically investigating the linkage between changing cocaine use and cognitive performance during a 1-year interval. Therefore, we categorized cocaine users in the Zurich Cocaine Cognition Study $\left(\mathrm{ZuCo}^{2} \mathrm{St}\right)$ as decreasers, stable users, or increasers after the 1-year follow-up. We then compared the course of cognitive performance between decreasers and increasers, whose test scores were normalized to the test-retest effects of a psychostimulantnaive control group that was also assessed twice. Because we were interested in the specific effects of cocaine, relatively pure users with little co-use of other illegal drugs were initially recruited. To objectively assess the initial severity and change in cocaine use and to control for co-use of other drugs, we performed quantitative hair and urine toxicology analyses at baseline and follow-up. Because we recently reported strong dose-response correlations between several cocaine use parameters and cognitive performance in cocaine users from the cross-sectional part of this study (Vonmoos et al, 2013), and based on previous animal studies suggesting that cognitive impairment in cocaine users might be drug-induced (Gould et al, 2012; Liu et al, 2008; Porter et al, 2011), we hypothesized that escalating cocaine use is associated with further cognitive impairment. Based on data suggesting that long-term cocaine abstinence of cocaine might be associated with partial recovery of neuropsychological performance (De Oliveira et al, 2009; Di Sclafani et al, 2002; van Gorp et al, 1999), we expect to find improved cognition in cocaine users with considerably decreased or ceased cocaine consumption.

\section{MATERIALS AND METHODS}

\section{Participants}

From a cross-sectional sample of 234 participants, 48 psychostimulant-naive controls and 57 cocaine users could be included in the longitudinal study (recruitment and selection details Supplementary Methods S1). At baseline, general exclusion criteria were neurological disorders or head injuries, severe somatic diseases, and any medication affecting the central nervous system. Controls were also excluded if they displayed current or previous DSM-IV Axis I psychiatric disorders (except for nicotine addiction), and regular illegal drug use ( $>15$ occasions lifetime, except for recreational cannabis use). Exclusion criteria for cocaine users were use of opioids, a polytoxic drug use pattern according to DSM-IV, and DSM-IV Axis I adult psychiatric disorders-except for cocaine, cannabis, nicotine, and alcohol abuse/dependence; history of affective disorders (current major depression was excluded); and attention deficit hyperactivity disorder (ADHD). Inclusion criteria for cocaine users were cocaine use of $>0.5 \mathrm{~g}$ per month, cocaine as primary drug, and an abstinence duration of $<6$ months at baseline.

Participants were asked to abstain from illegal substances for at least $72 \mathrm{~h}$ and from alcohol for $24 \mathrm{~h}$ before test sessions. Compliance with these instructions was controlled using urine screenings (semi-quantitative enzyme multiplied immunoassay method, for technical details see Vonmoos et al, 2013). Drug use severity was assessed by 6-month hair toxicology analyses (liquid chromatographytandem mass spectrometry; Vonmoos et al, 2013). The study was approved by the Cantonal Ethics Committee of Zurich. All participants provided written informed consent and received compensation for their participation.

\section{Group Assignment}

The decisive criterion for replicable group assignment was a combination of absolute and relative changes in cocaine concentration in hair samples between baseline ( $\mathrm{t} 1$ ) and followup ( $\mathrm{t} 2$ ). The absolute criterion was based on a shift in cocaine concentration of at least $0.5 \mathrm{ng} / \mathrm{mg}$, according to a commonly accepted cutoff value for reliably detection of cocaine use (Bush, 2008; Cooper et al, 2012). The relative criterion was based on a minimal increase of $20 \%$ or a minimal decrease of $10 \%$ in the robust hair toxicology parameter cocaine $_{\text {total }}(=$ cocaine + benzoylecgonine + norcocaine) (Hoelzle et al, 2008). According to these criteria, cocaine users were divided into three groups of similar size: 19 cocaine increasers consumed substantially more cocaine at follow-up (mean increase $+30.4 \mathrm{ng} / \mathrm{mg}$ (297\%), range $0.5-268.5 \mathrm{ng} / \mathrm{mg}$ (20-5374\%), SD $61.9 \mathrm{ng} / \mathrm{mg}$ ), whereas 19 cocaine decreasers consumed substantially less cocaine (mean decrease $-10.6 \mathrm{ng} / \mathrm{mg}(-72 \%)$, range from -116.9 to $-0.6 \mathrm{ng} / \mathrm{mg}(-100$ to $-12 \%)$, SD $26.7 \mathrm{ng} / \mathrm{mg}$ ), and 19 users with a relatively stable cocaine use pattern did not meet both criteria, and, thus, were not further analyzed (Supplementary Figure S1).

\section{Procedure}

The test procedure was similar in baseline and follow-up. Trained psychologists conducted the Structured Clinical Interview for DSM-IV (American Psychiatric Association, 
1994). Drug use was assessed with a structured and standardized interview for Psychotropic Drug Consumption (Quednow et al, 2004). Cognitive performance was assessed with a neuropsychological test battery comprising three tasks of the Cambridge Neuropsychological Test Automated Battery (CANTAB, www.cantab.com): Rapid Visual Processing, Spatial Working Memory, Paired Associates Learning (PAL); a German version of the Rey Auditory Verbal Learning Test (RAVLT)(Helmstaedter et al, 2001); and the Letter Number Sequencing Task (LNST)(Wechsler, 1997). At follow-up, parallel test-versions were used for the PAL, RAVLT, and LNST. In contrast to the cross-sectional analysis, we excluded the CANTAB Intra/Extradimensional Set Shifting from the longitudinal analysis because of an evident ceiling effect at baseline (Vonmoos et al, 2013).

Analogous to the cross-sectional part of the study (Vonmoos et al, 2013), 13 predefined main cognitive test parameters were $z$-transformed on the basis of means and SDs of the control group $(n=48)$ at baseline. If necessary, test scores were reversed so that high scores always indicated better cognitive performance. Test parameters were reduced to four cognitive domains (attention, working memory, declarative memory, and executive functions, for details see Supplementary Methods S2). Furthermore, the four $z$-scored domains were equally integrated into a broad global cognitive index (GCI).

\section{Statistical Analysis}

Statistical analyses were performed with SPSS Statistics 19.0 (IBM, Zurich, Switzerland). Effect sizes were calculated in SPSS and with $\mathrm{G}^{\star}$ Power 3.1 (Faul et al, 2007). Frequency data were analyzed by means of Pearson's $\chi^{2}$ test. Group differences in cognitive test scores at baseline and follow-up were analyzed by analyses of variance (ANOVA). For the longitudinal analysis, cocaine user groups and subgroups were analyzed using mixed design analyses of covariance (ANCOVA), omnibus tests (group*time) were followed by Sidakcorrected pairwise pre-post comparisons adjusted for testretest effects. Because ADHD has previously been linked to both, cognitive performance in cocaine users (Preller et al, 2014, Vonmoos et al, 2013) and substance use in general (Wilson, 2007), mixed design analyses were corrected for ADHD as measured by the ADHD Self-Rating scale (Roesler et al, 2004). Given that we expected inevitable test-retest effects in all groups and because we aimed to estimate the change of the cocaine using groups relative to the control group, in which the general cognitive performance should be constant across 1 year, we corrected the user groups' change scores by subtracting the mean change score of the control group. To relate cognitive change scores to varying cocaine use during the test interval, Pearson product-moment correlation analyses (two-tailed) were conducted in the cocaine user group. The confirmatory statistical comparisons were carried out on a significance level of $p<0.05$ (two-tailed).

\section{RESULTS}

\section{Demographic Characteristics and Drug Use}

Controls, increasers, and decreasers did not differ regarding demographic data and time interval between baseline and follow-up (Table 1, for details considering the not further analyzed group of stable cocaine users, see Supplementary Table S1). However, as previously shown, both cocaine user groups displayed significantly higher BDI and ADHD-selfreport sum scores than controls (Vonmoos et al, 2013). Hair samples and cumulative doses revealed a clear dominance of cocaine compared with other illegal drugs, as intended by the inclusion and exclusion criteria. At baseline, increasers and decreasers displayed similar cocaine hair concentrations; however, at follow-up increasers showed an $\sim 10$-fold higher concentration of cocaine than decreasers. Whereas hair analyses for increasers showed a threefold increase between baseline and follow-up, decreasers displayed only

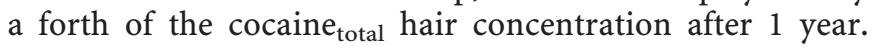
In contrast to baseline, none of the self-reported cocaine use parameters correlated with hair cocaine concentrations in the follow-up $(r=0.02$ to $-0.29, p=0.89$ to 0.08$)$, highlighting the importance of objective drug use measures in longitudinal studies (Supplementary Table S2).

\section{Test Scores at Baseline}

As previously demonstrated in the cross-sectional sample of this study (Vonmoos et al, 2013), ANOVAs showed significant group effects for the GCI, both memory domains, and the executive function but only a statistical trend for attention (Table 2, for details regarding single test parameters, see Supplementary Table S3), indicating moderate to strong cognitive impairments in both cocaine user groups compared with controls (Cohen's $d=0.47-0.79$ ). Increasers and decreasers did not substantially differ in the GCI $(p=0.99, d=0.08)$ and all four domains $(p \geqslant 0.94, d \leqslant 0.14)$ at baseline.

\section{Change between Baseline and Follow-Up}

Because of strong test-retest effects, at the follow-up, all groups displayed a better performance on the GCI, all domains, and the majority of single tests compared with baseline (Table 2, Supplementary Table S3). Of note, testretest improvements in controls and decreasers were substantially stronger than in the increaser group.

An ADHD-corrected mixed ANCOVA for the two user groups revealed a significant group ${ }^{\star}$ time interaction effect on working memory $\left(\mathrm{F}_{1,35}=4.85, p<0.05,{ }_{p} \eta^{2}=0.12\right)$ (Figure 1). Furthermore, there was a non-significant trend for a group ${ }^{\star}$ time interaction in the GCI $\left(\mathrm{F}_{1,35}=2.96\right.$, $p=0.09,{ }_{p} \eta^{2}=0.08$ )(for a GCI analysis including the group of stable cocaine users, see Supplementary Figure S2). However, the effect sizes of the group ${ }^{\star}$ time interactions regarding declarative memory $\left(\mathrm{F}_{1,35}=2.11, p=0.16\right.$, $\left.{ }_{p} \eta^{2}=0.06\right)$, attention $\left(\mathrm{F}_{1,35}=0.73, p=0.40,{ }_{p} \eta^{2}=0.02\right)$, and executive functions $\left(\mathrm{F}_{1,35}=0.15, p=0.70,{ }_{p} \eta^{2}=0.004\right)$ were rather small.

In subsequent pairwise Sidak pre-post comparisons adjusted for test-retest effects (Figure 1, Supplementary Table S4), increasers showed a significant cognitive decline in working memory $(p<0.05, d=-0.52)$. Additional exploratory analysis revealed small effect sizes for a decline in increasers with regard to declarative memory $(d=-0.16)$, attention $(d=-0.04)$, and GCI $(d=-0.21)$. By contrast, performance improvements of the decreasers 
Table I Demographic Data and Pattern of Substance Use

\begin{tabular}{|c|c|c|c|c|c|c|c|c|c|c|c|c|c|c|}
\hline & \multicolumn{7}{|c|}{ Baseline ( $t \mathrm{l})$} & \multicolumn{7}{|c|}{ I-year follow-up (t2) ${ }^{a}$} \\
\hline & $\begin{array}{l}\text { Controls } \\
(n=48)\end{array}$ & $\begin{array}{c}\text { Cocaine } \\
\text { increaser } \\
(n=19)\end{array}$ & $\begin{array}{c}\text { Cocaine } \\
\text { decreaser } \\
(n=19)\end{array}$ & $\mathbf{F} / \chi^{2} / \mathbf{T}$ & d.f., d.f.err & $p$ & $\begin{array}{l}\text { Effect } \\
\text { size }\end{array}$ & $\begin{array}{l}\text { Controls } \\
(n=48)\end{array}$ & $\begin{array}{c}\text { Cocaine } \\
\text { increaser } \\
(n=19)\end{array}$ & $\begin{array}{c}\text { Cocaine } \\
\text { decreaser } \\
(n=19)\end{array}$ & $\mathbf{F} / \chi^{2} / \mathbf{T}$ & $d f, f_{e r r}$ & $p$ & $\begin{array}{l}\text { Effect } \\
\text { size }\end{array}$ \\
\hline Age, years & $30.3(8.9)$ & $31.5(9.4)$ & $31.4(8.3)$ & $0.20^{\mathrm{b}}$ & 2,83 & 0.82 & ${ }_{p} \eta^{2}=0.00$ & & & & & & & \\
\hline Sex (female/male) & $16 / 32$ & $3 / 16$ & $5 / 14$ & $2.11^{c}$ & 2 & 0.35 & $V=0.16$ & & & & & & & \\
\hline Verbal IQ (MWT-B) ${ }^{d}$ & $107.6(10.0)$ & $102.9(9.7)$ & $103.8(7.1)$ & $2.20^{\mathrm{b}}$ & 2,83 & 0.12 & ${ }_{p} \eta^{2}=0.05$ & & & & & & & \\
\hline Education, years & $10.8(1.8)$ & $10.4(1.8)$ & $10.0(1.5)$ & $1.30^{\mathrm{b}}$ & 2,83 & 0.28 & $p \eta^{2}=0.03$ & & & & & & & \\
\hline ADHD-SR score (0-22) & $7.7(5.2)$ & I $3.5(9.4)^{* * *}$ & |4.| (6.8)*** & $8.83^{\mathrm{b}}$ & 2,83 & $<0.001$ & ${ }_{p} \eta^{2}=0.18$ & & & & & & & \\
\hline ADHD DSM-IV $(y / n)^{e}$ & $0 / 48$ & $4 / 15$ & $3 / 16$ & $7.02^{c}$ & 2 & 0.03 & $V=0.28$ & & & & & & & \\
\hline Weeks between $\mathrm{t} I$ and $\mathrm{t} 2$ & $58.2(10.1)$ & $59.3(12.1)$ & $61.9(14.5)$ & $.69^{\mathrm{b}}$ & 2,83 & 0.50 & ${ }_{p} \eta^{2}=0.02$ & & & & & & & \\
\hline BDI score $(0-63)$ & $3.5(3.3)$ & $7.3(8.0) *$ & $8.7(6.5) * *$ & $7.53^{\mathrm{b}}$ & 2,83 & $<0.001$ & $\eta^{2}=0.15$ & & & & & & & \\
\hline BDI depression $(y / n)^{f}$ & $0 / 48$ & $1 / 18$ & $1 / 18$ & $2.59^{c}$ & 2 & 0.27 & $V=0.17$ & & & & & & & \\
\hline \multicolumn{15}{|l|}{ Cocaine } \\
\hline Times per week ${ }^{g}$ & - & $1.6(1.8)$ & $1.0(1.3)$ & $1.17^{\mathrm{h}}$ & 36 & 0.25 & $d=0.38$ & - & I.I (0.8) & $0.3(0.3)$ & $3.85^{h}$ & 36 & $<0.001$ & $d=1.32$ \\
\hline Grams per week ${ }^{g}$ & - & $2.0(2.5)$ & $1.7(2.3)$ & $0.41^{\mathrm{h}}$ & 36 & 0.68 & $d=0.12$ & - & $1.6(2.5)$ & $0.4(0.4)$ & $2.18^{\mathrm{h}}$ & 36 & 0.04 & $d=0.67$ \\
\hline Years of use & - & $7.0(5.5)$ & $8.2(5.4)$ & $0.68^{h}$ & 36 & 0.50 & $d=0.22$ & - & $8.9(5.4)$ & $9.7(5.2)$ & $0.45^{\mathrm{h}}$ & 36 & 0.65 & $d=0.15$ \\
\hline Maximum dose $(\mathrm{g} / \mathrm{day})^{i}$ & - & $4.7(4.4)$ & $5.9(6.4)$ & $0.71^{\mathrm{h}}$ & 36 & 0.48 & $d=0.22$ & - & $3.7(2.5)$ & $3.1(2.8)$ & $0.63^{h}$ & 36 & 0.53 & $d=0.23$ \\
\hline Cumulative dose $(\mathrm{g})^{i}$ & - & $1182(1635)$ & $3698(8585)$ & $1.25^{\mathrm{h}}$ & 36 & 0.22 & $d=0.4 \mathrm{I}$ & - & $91(119)$ & $49(89)$ & $1.25^{\mathrm{h}}$ & 36 & 0.22 & $d=0.40$ \\
\hline Last consumption (days) & - & $18.5(25.1)$ & $20.8(22.2)$ & $0.29^{h}$ & 36 & 0.77 & $d=0.08$ & - & $7.0(6.3)$ & $81.4(145.1)$ & $2.23^{\mathrm{h}}$ & 36 & 0.03 & $d=0.72$ \\
\hline Cocaine craving $(0-70)^{j}$ & - & $19.8(9.5)$ & $17.7(7.2)$ & $0.79^{h}$ & 36 & 0.44 & $d=0.25$ & - & $20.5(10.8)$ & $15.8(6.2)$ & $1.66^{\mathrm{h}}$ & 36 & 0.11 & $d=0.53$ \\
\hline \multicolumn{15}{|l|}{ Hair analysis $(n g / m g)^{k}$} \\
\hline Cocaine $_{\text {total }}$ & - & $10.3(29.2)$ & $14.9(32.2)$ & $0.46^{h}$ & 36 & 0.65 & $d=0.15$ & - & $40.7(76.1)$ & $4.2(8.2)$ & $2.08^{h}$ & 36 & 0.05 & $d=0.67$ \\
\hline Cocaine & - & $8.2(23.3)$ & I $1.4(23.9)$ & $0.42^{h}$ & 36 & 0.68 & $d=0.14$ & - & $31.7(56.5)$ & $3.1(5.9)$ & $2.19^{h}$ & 36 & 0.03 & $d=0.71$ \\
\hline Benzoylecgonine & - & $1.9(5.5)$ & $3.1(7.6)$ & $0.58^{h}$ & 36 & 0.56 & $d=0.18$ & - & $8.3(19.6)$ & $1.0(2.2)$ & $1.62^{\mathrm{h}}$ & 36 & 0.11 & $d=0.52$ \\
\hline Cocaethylene & - & $1.0(2.8)$ & $0.9(2.8)$ & $0.11^{\mathrm{h}}$ & 36 & 0.91 & $d=0.04$ & - & $1.2(2.1)$ & $0.3(1.0)$ & $1.56^{\mathrm{h}}$ & 36 & 0.13 & $d=0.55$ \\
\hline Norcocaine $_{t}$ & - & $0.2(0.5)$ & $0.4(0.8)$ & $0.83^{h}$ & 36 & 0.41 & $d=0.30$ & - & $0.6(1.4)$ & $0.1(0.1)$ & $1.71^{\mathrm{h}}$ & 36 & 0.10 & $d=0.50$ \\
\hline Urine toxicology $(n / p)^{i}$ & $48 / 0$ & $14 / 5$ & $16 / 3$ & $0.63^{c}$ & । & 0.43 & $V=0.13$ & $48 / 0$ & $7 / 12$ & $18 / 1$ & $14.15^{c}$ & I & $<0.001$ & $V=0.61$ \\
\hline \multicolumn{15}{|l|}{ Alcohol } \\
\hline Grams per week ${ }^{g}$ & I 19.9 (136.8) & $169.4(129.2)$ & I55.3 (| 46.4$)$ & $1.07^{\mathrm{b}}$ & 2,83 & 0.35 & $p \eta^{2}=0.03$ & $104.3(88.6)$ & $259.7(244.5)^{* * * *}$ & $127.4(141.4)^{\circ}$ & $7.71^{\mathrm{b}}$ & 2,83 & $<0.001$ & ${ }_{p} \eta^{2}=0.16$ \\
\hline Years of use & |3.3(8.8) & | $3.7(7.6)$ & $12.0(7.3)$ & $0.23^{b}$ & 2,83 & 0.79 & $p \eta^{2}=0.01$ & | $4.0(8.7)$ & $14.8(7.5)$ & $12.6(7.9)$ & $0.34^{\mathrm{b}}$ & 2,83 & 0.71 & ${ }_{p} \eta^{2}=0.01$ \\
\hline \multicolumn{15}{|l|}{ Nicotine } \\
\hline Smoking $(y / n)^{\prime}$ & $37 / 11$ & $14 / 5$ & $14 / 5$ & $0.13^{c}$ & 2 & 0.94 & $V=0.04$ & $40 / 8$ & $15 / 4$ & $13 / 6$ & $1.83^{c}$ & 2 & 0.40 & $V=0.15$ \\
\hline Cigarettes per dayg & $8.7(8.7)$ & $12.8(11.2)$ & $9.5(8.2)$ & $1.38^{\mathrm{b}}$ & 2,83 & 0.26 & ${ }_{p} \eta^{2}=0.03$ & $8.2(8.7)$ & $13.4(12.0)$ & $8.2(7.8)$ & $2.31^{\mathrm{b}}$ & 2,83 & 0.11 & ${ }_{p} \eta^{2}=0.05$ \\
\hline Years of use & $9.3(8.3)$ & $10.4(8.9)$ & $12.7(10.3)$ & $0.95^{\mathrm{b}}$ & 2,83 & 0.39 & ${ }_{p} \eta^{2}=0.02$ & $10.5(8.8)$ & I2.5 (8.6) & $12.6(9.9)$ & $0.56^{\mathrm{b}}$ & 2,83 & 0.57 & ${ }_{p} \eta^{2}=0.01$ \\
\hline \multicolumn{15}{|l|}{ Cannabis } \\
\hline Grams per week ${ }^{g}$ & $0.6(1.6)$ & $3.3(8.9)$ & $1.2(2.3)$ & $2.38^{b}$ & 2,83 & 0.10 & ${ }_{p} \eta^{2}=0.05$ & $0.5(1.6)$ & $2.1(4.6)$ & I.I (2.7) & $2.28^{\mathrm{b}}$ & 2,83 & 0.11 & $p \eta^{2}=0.05$ \\
\hline Years of use & $4.5(4.9)$ & $9.5(8.5)^{*}$ & $10.1(9.7)^{*}$ & $5.92^{\mathrm{b}}$ & 2,83 & 0.004 & $p \eta^{2}=0.12$ & $4.6(5.9)$ & $10.5(9.8)^{*}$ & $8.6(9.7)$ & $4.64^{\mathrm{b}}$ & 2,83 & 0.01 & ${ }_{p} \eta^{2}=0.10$ \\
\hline Cumulative dose (grams) & 980 (3985) & $3199(5899)$ & 2606 (6359) & $1.61^{\mathrm{b}}$ & 2,83 & 0.21 & ${ }_{p} \eta^{2}=0.04$ & $53.4(180)$ & $217.8(526.5)$ & $84.7(189.6)$ & $2.15^{b}$ & 2,83 & 0.12 & ${ }_{p} \eta^{2}=0.05$ \\
\hline Last consumption (days) ${ }^{\mathrm{m}}$ & $39.3(54.8) ; n=22$ & $10.0(9.9) ; n=14$ & $25.4(32.8) ; n=12$ & $2.19^{\mathrm{b}}$ & 2,45 & 0.12 & $p \eta^{2}=0.09$ & $36.5(77.5) ; n=22$ & $9.7(24.6) ; n=13$ & $50.8(74.5) ; n=10$ & $1.20^{\mathrm{b}}$ & 2,42 & 0.31 & ${ }_{p} \eta^{2}=0.05$ \\
\hline Urine toxicology $(n / p)^{i}$ & $42 / 6$ & $15 / 4$ & $14 / 5$ & $2.03^{c}$ & 2 & 0.36 & $V=0.15$ & $42 / 6$ & $7 / 12$ & $15 / 4$ & $18.61^{\mathrm{c}}$ & 2 & $<0.001$ & $V=0.47$ \\
\hline
\end{tabular}


Table I (Continued)

\begin{tabular}{|c|c|c|c|c|c|c|c|c|c|c|c|c|c|c|}
\hline & \multicolumn{7}{|c|}{ Baseline $(t \mathrm{l})$} & \multicolumn{7}{|c|}{ I-year follow-up (t2) ${ }^{a}$} \\
\hline & $\begin{array}{l}\text { Controls } \\
(n=48)\end{array}$ & $\begin{array}{c}\text { Cocaine } \\
\text { increaser } \\
(n=19)\end{array}$ & $\begin{array}{c}\text { Cocaine } \\
\text { decreaser } \\
(n=19)\end{array}$ & $\mathbf{F} / \chi^{2} / \mathbf{T}$ & d.f., d.f.err & $p$ & $\begin{array}{l}\text { Effect } \\
\text { size }\end{array}$ & $\begin{array}{l}\text { Controls } \\
(n=48)\end{array}$ & $\begin{array}{c}\text { Cocaine } \\
\text { increaser } \\
(n=19)\end{array}$ & $\begin{array}{c}\text { Cocaine } \\
\text { decreaser } \\
(n=19)\end{array}$ & $\mathbf{F} / \chi^{2} / \mathbf{T}$ & $d f, d f_{\text {err }}$ & $p$ & $\begin{array}{l}\text { Effect } \\
\text { size }\end{array}$ \\
\hline \multicolumn{15}{|l|}{ Amphetamine } \\
\hline Grams per week ${ }^{g}$ & $0.0(0.1)$ & $0.1(0.1)^{* * *}$ & $0.0(0.1)$ & $5.18^{\mathrm{b}}$ & 2,83 & 0.008 & $p \eta^{2}=0.11$ & $0.0(0.0)$ & $0.1(0.2) * *$ & $0.0(0.1)$ & $5.89^{b}$ & 2,83 & 0.004 & ${ }_{p} \eta^{2}=0.12$ \\
\hline Years of use & $0.0(0.0)$ & $3.3(4.0)^{* * * * * *}$ & $1.3(3.1)^{\circ}$ & $13.73^{b}$ & 2,83 & $<0.001$ & ${ }_{p} \eta^{2}=0.25$ & $0.1(0.5)$ & $3.2(4.9)^{* * *}$ & $2.7(5.5)^{*}$ & $7.46^{\mathrm{c}}$ & 2,83 & 0.001 & ${ }_{p} \eta^{2}=0.15$ \\
\hline Cumulative dose (g) & $0.0(0.1)$ & $56.0(177.6)^{*}$ & $16.2(35.9)$ & $2.99^{\mathrm{b}}$ & 2,83 & 0.06 & ${ }_{p} \eta^{2}=0.07$ & $0.0(0.1)$ & $4.4(8.9)^{* * *}$ & $1.4(3.5)$ & $6.47^{b}$ & 2,83 & 0.002 & ${ }_{p} \eta^{2}=0.13$ \\
\hline Last consumption (days) ${ }^{\mathrm{m}}$ & $121.6(-) ; n=1$ & $73.6(61.6) ; n=10$ & $90.9(80.6) ; n=3$ & $0.29^{b}$ & 2,11 & 0.75 & ${ }_{p} \eta^{2}=0.05$ & $17.5(-) ; n=1$ & $35.7(31.5) ; n=8$ & $99.8(108.1) ; n=4$ & $1.48^{\mathrm{b}}$ & 2,10 & 0.27 & ${ }_{p} \eta^{2}=0.23$ \\
\hline Hair analysis $(\mathrm{ng} / \mathrm{mg})$ & $0.0(0.0)$ & $0.1(0.2)^{*}$ & $0.0(0.0)$ & $4.35^{b}$ & 2,83 & 0.02 & ${ }_{p} \eta^{2}=0.09$ & $0.0(0.0)$ & $0.1(0.2)$ & $0.1(0.2)$ & $2.89^{b}$ & 2,83 & 0.06 & ${ }_{p} \eta^{2}=0.07$ \\
\hline \multicolumn{15}{|l|}{ MDMA } \\
\hline Tablets per week ${ }^{\mathrm{g}}$ & $0.0(0.0)$ & $0.0(0.1)^{* * * * *}$ & $0.0(0.0)^{\circ}$ & $7.42^{\mathrm{b}}$ & 2,83 & 0.001 & ${ }_{p} \eta^{2}=0.15$ & $0.0(0.0)$ & $0.4(0.9) * *$ & $0.0(0.0)^{\circ}$ & $5.54^{\mathrm{b}}$ & 2,83 & 0.006 & ${ }_{p} \eta^{2}=0.12$ \\
\hline Years of use & $0.3(1.0)$ & $3.5(4.5)^{* * * *}$ & $2.4(4.6) *$ & $8.42^{b}$ & 2,83 & $<0.001$ & $p \eta^{2}=0.17$ & $0.2(1.4)$ & $3.8(5.5) * *$ & $3.2(5.6) *$ & $7.78^{\mathrm{b}}$ & 2,83 & $<0.001$ & ${ }_{p} \eta^{2}=0.16$ \\
\hline Cumulative dose (tablets) & $1.3(4.0)$ & $108.8(249.7)^{* *}$ & | $8.7(46.2)$ & $5.71^{b}$ & 2,83 & 0.005 & ${ }_{p} \eta^{2}=0.12$ & $0.2(0.8)$ & $17.0(49.3)^{*}$ & $2.8(5.2)$ & $3.67^{b}$ & 2,83 & 0.03 & ${ }_{p} \eta^{2}=0.08$ \\
\hline Last consumption (days) ${ }^{\mathrm{m}}$ & $5.0(-) ; n=1$ & $89.9(64.6) ; n=7$ & $40.2(34.4) ; n=4$ & $1.63^{b}$ & 2,9 & 0.25 & $p \eta^{2}=0.27$ & $91.2(30.4) ; n=3$ & $41.6(54.8) ; n=6$ & $47.8(47.8) ; n=5$ & $1.11^{\mathrm{b}}$ & 2,11 & 0.36 & ${ }_{p} \eta^{2}=0.17$ \\
\hline Hair analysis (ng/mg) & $0.0(0.0)$ & $0.3(0.7)$ & $0.4(1.5)$ & $2.23^{b}$ & 2,83 & 0.11 & $p \eta^{2}=0.05$ & $0.0(0.0)$ & $0.5(0.8)^{* * * * *}$ & $0.1(0.3)$ & $7.87^{b}$ & 2,83 & $<0.001$ & ${ }_{p} \eta^{2}=0.16$ \\
\hline \multicolumn{15}{|l|}{$G H B$} \\
\hline Cumulative dose (pipettes) & $0.0(0.0)$ & $0.5(0.7)$ & $0.5(1.7)$ & $3.36^{\mathrm{b}}$ & 2,83 & 0.04 & ${ }_{p} \eta^{2}=0.07$ & $0.0(0.0)$ & $0.0(0.0)$ & $0.0(0.0)$ & - & - & - & - \\
\hline \multicolumn{15}{|l|}{ Hallucinogens } \\
\hline Cumulative dose (times) & $0.9(2.2)$ & $27.9(72.8)^{*}$ & $9.9(22.9)$ & $3.92^{b}$ & 2,83 & 0.02 & ${ }_{p} \eta^{2}=0.09$ & $0.0(0.0)$ & $1.1(1.6)^{* * * * *}$ & $0.6(1.5)$ & $8.57^{b}$ & 2,83 & $<0.001$ & ${ }_{p} \eta^{2}=0.17$ \\
\hline \multicolumn{15}{|l|}{ Methlyphenidate } \\
\hline Cumulative dose (tablets) & $0.0(0.0)$ & $20.2(60.4)^{*}$ & $0.5(2.3)$ & $3.76^{\mathrm{b}}$ & 2,83 & 0.03 & ${ }_{p} \eta^{2}=0.08$ & $0.0(0.1)$ & $67.7(239.5)$ & $0.3(0.6)$ & $2.72^{b}$ & 2,83 & 0.07 & ${ }_{p} \eta^{2}=0.06$ \\
\hline Hair analysis (ng/mg) & $0.0(0.0)$ & $0.0(0.1)$ & $0.0(0.0)$ & $1.80^{\mathrm{b}}$ & 2,83 & 0.17 & $p \eta^{2}=0.04$ & $0.0(0.0)$ & $0.1(0.2)^{*}$ & $0.0(0.0)$ & $3.62^{b}$ & 2,83 & 0.03 & ${ }_{p} \eta^{2}=0.08$ \\
\hline
\end{tabular}

Means and SD. Significant $p$ values are shown in bold.

aParameters at follow-up refer to the I-year period between $\mathrm{tl}$ and $\mathrm{t} 2$

${ }^{b}$ ANOVA (all groups, with significant Sidak post hoc test vs control group: ${ }^{*} p<0.05$; $* * * 0.0$ I; $* * * * 0<0.00$ I; vs cocaine increaser: ${ }^{\circ} p<0.05$ ).

${ }^{c} \chi^{2}$-test (all groups/cocaine users only) for frequency data.

¿Verbal IQ was assessed by the Mehrfachwahl Wortschatz Intelligenztest (Lehrl, 1999).

eADHD-SR, ADHD self-rating scale (cutoff DSM-IV criteria) (Roesler et al, 2004).

${ }^{\mathrm{f} B D I}$, Beck Depression Inventory (cutoff $\geqslant 18$ ) (Hautzinger et al, 1994).

${ }^{8}$ Average use during the last 6 months.

hindependent $t$-test (cocaine users only).

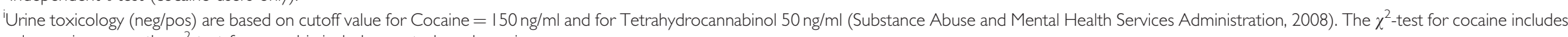
only cocaine users, the $\chi^{2}$-test for cannabis includes controls and cocaine users.

${ }^{j}$ Craving for cocaine was assessed by the Brief-CCQ (Sussner et al, 2006).

${ }^{k}$ Hair samples were voluntary and data are missing for three controls.

'Smoking habits were assessed by the Fagerstroem Test of Nicotine Dependence (Heatherton et al, 1991).

mLast consumption is averaged only for persons who used the drug in the last 6 months. 
were not significant but revealed small to moderate effect sizes in attention $(d=0.22)$, working memory $(d=0.21)$, declarative memory $(d=0.30)$, and the GCI $(d=0.33)$.

In addition, correlation analyses within a consolidated group of increasers and decreasers indicated a significant association between cumulative cocaine dose used during the test interval and change scores in attention $(r=0.34$, $p<0.05)$ as well as a significant relation between changes in the hair parameter cocaine total $_{\text {and }}$ change scores in the declarative memory $(r=0.39, p<0.05)$ (for details see Supplementary Table S5).

As we have previously shown that age of onset was an important modulator of cognitive performance in cocaine users (Vonmoos et al, 2013), we further investigated whether age of onset was linked to the significantly different change in working memory of cocaine increasers and decreasers during the test interval. Although the increasers did not show any substantial correlation between age of onset and working memory change score $(r=-0.10, p=0.68)$, there was a significant association in cocaine decreasers $(r=0.54, p<0.05)$, indicating that early onset of cocaine use goes along with reduced recovery of working memory when cocaine use is considerably reduced (Figure 2).

To analyze whether decreasers recover depending on their initial level of cocaine use, we correlated their cocaine use levels at baseline (hair concentration cocaine total $_{\text {) with }}$ the cognitive change scores. However, we did not find a significant correlation in the GCI $(r=-0.10, p=0.68$, $n=19)$ or any other domain ( $r=-0.35$ to $0.18, p=0.14-$ $0.47, n=19)$.

\section{Test Scores at Follow-Up}

In contrast to baseline, decreasers performed slightly, albeit non-significantly better than increasers on the GCI $(d=0.37)$, all domains $(d=0.14-0.42)$, and each single parameter $(d=0.14-0.49)$ (Table 2). Accordingly, the domain differences between decreasers and controls were reduced to non-significant small to moderate effect sizes $(d=0.24-0.59)$. Controls and increasers still differed significantly in the GCI $(d=0.85)$, working memory $(d=0.95)$, and declarative memory $(d=0.78)$.

\section{Impact of Ceased and Strongly Intensified Cocaine Use}

To investigate the impact of ceased or strongly intensified cocaine use, we split cocaine increaser (low/high; cutoff $\Delta_{\mathrm{t} 2 \text {-t1 }}$ cocaine $_{\text {total }}=10 \mathrm{ng} / \mathrm{mg}$ ), and decreaser subgroups (ceased use/ongoing use; cutoff cocaine total ceased use at follow-up $<0.5 \mathrm{ng} / \mathrm{mg}$ )(for a detailed subgroup description, see Supplementary Table S6). Given the lack of power in such a four-group comparison, the mixed ANCOVA (corrected for ADHD) displayed only non-significant group*time interactions regarding all domains, but with some interesting effect sizes: GCI $\left(F_{3,33}=1.70, \quad p=0.19, p \eta^{2}=0.13\right)$, attention $\left(F_{3,33}=2.09, \quad p=0.12, \quad p \eta^{2}=0.16\right), \quad$ working memory $\left(F_{3,33}=1.89, \quad p=0.15, \quad p \eta^{2}=0.15\right), \quad$ declarative memory $\left(F_{3,33}=1.69, p=0.19,{ }_{p} \eta^{2}=0.13\right)$, and executive functions $\left(F_{3,33}=0.22, p=0.88, p \eta^{2}=0.02\right)$. As we were specifically interested in whether long-term cocaine abstinence has an effect on cognition, we interpreted Sidak pre-post comparisons in the group of ceasing cocaine users. Notably, users 

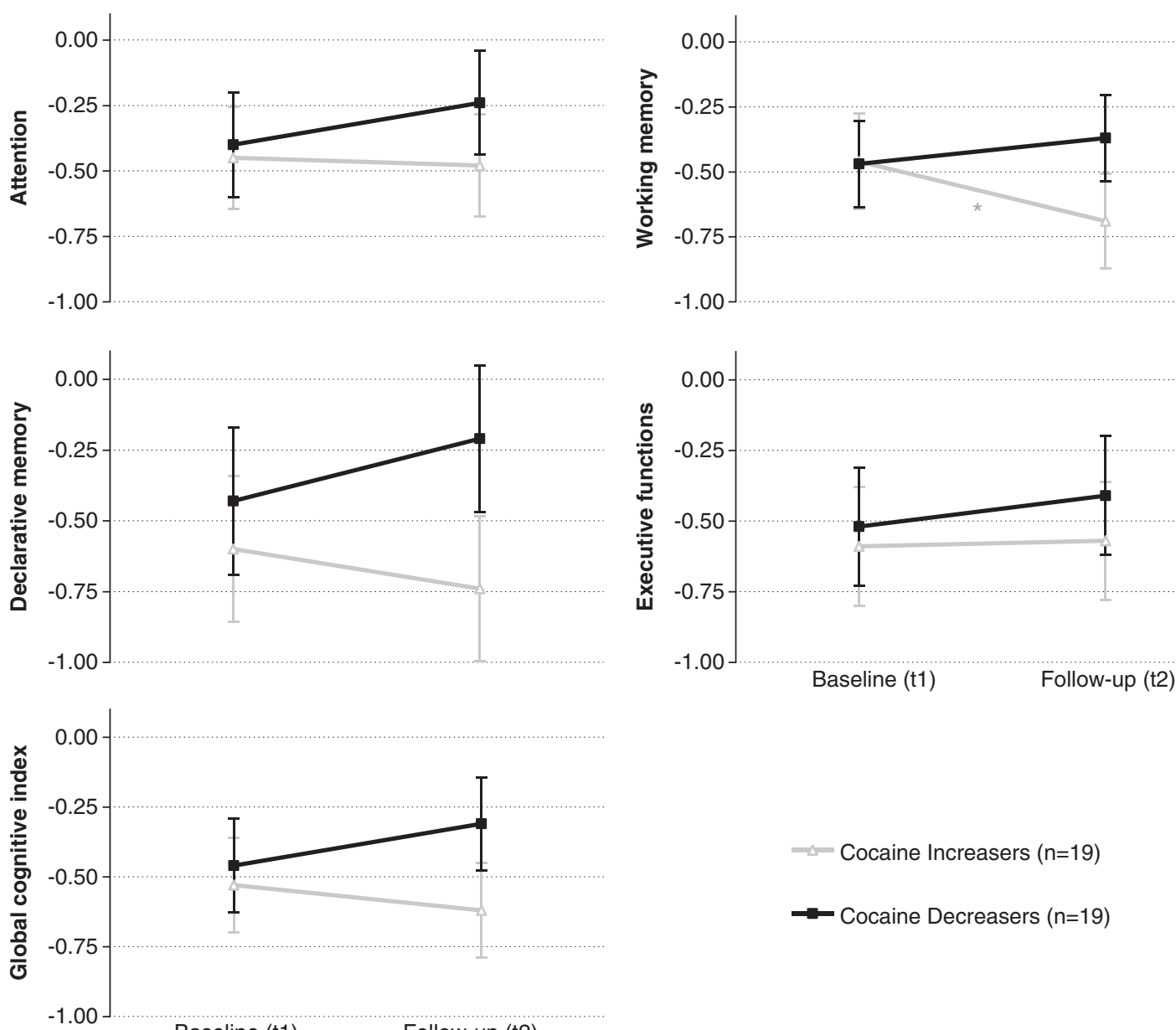

Baseline (t1)

Follow-up (t2)

Figure I Development of cognitive functioning in cocaine increasers and decreasers within I year. Z-scores and SEM for the cognitive domains (corrected for ADHD). Z-score transformation was based on control group mean and SD at baseline. Follow-up values were adjusted for the test-retest effect. Change in cognitive test performance from the baseline ( $\mathrm{tI})$ to the I-year follow-up (t2): Sidak post hoc tests $(* p<0.05)$. GCl: increaser $p=0.36$, $d=-0.21$, decreaser $p=0.14, d=0.33$. Attention: increaser $p=0.87, d=-0.04$, decreaser $p=0.30, d=0.22$. Working memory: increaser $p<0.05$, $d=-0.52$, decreaser $p=0.34, d=0.21$. Declarative memory: increaser $p=0.44, d=-0.16$, decreaser $p=0.21, d=0.30$. Executive functions: increaser $p=0.92, d=0.02$, decreaser $p=0.52, d=0.14$.

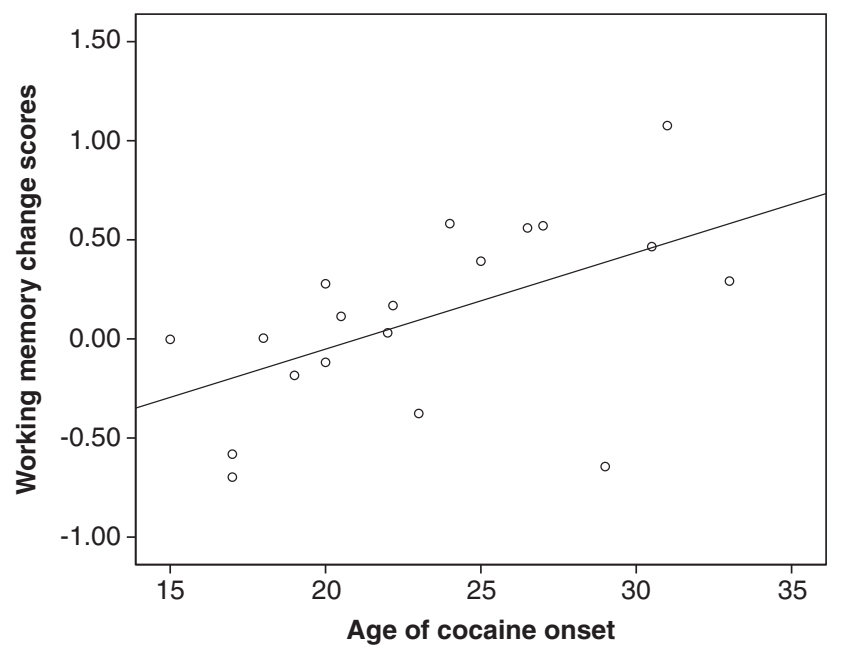

Figure 2 Correlation of age of cocaine use onset and working memory change scores $\left(\Delta_{t 2-t 1}\right)$ in decreasing cocaine users $(n=19)$. Pearson product-moment correlation (two-tailed): $r=0.54, p<0.05$. Working memory change scores were adjusted for the test-retest effect. who completely stopped cocaine use for at least 6 months (negative hair toxicology) displayed a significantly improved GCI $(p<0.05, d=0.93)$, attention $(p<0.05, d=1.10)$, and declarative memory $(p<0.05, d=0.65)$, resulting in follow-up test scores in the range of the control group (Figure 3).

An ADHD-corrected mixed ANCOVA of the GCI including only cocaine increasers stratified according to positive $(n=12)$ and negative cocaine urine toxicologies $(n=7)$ at baseline, and follow-up did not reveal a significant group ${ }^{*}$ time interaction $\left(F_{1,16}=0.00, p=0.99, p \eta^{2}=0.00\right)$, indicating that recent cocaine use likely did not explain the decline in test performance in increasers (Supplementary Figure S3). Because only one cocaine decreaser featured a positive urine toxicology analysis at the follow-up, we did not analyze the group of decreasers further.

\section{DISCUSSION}

This longitudinal study is the first linking objectively quantified changes in cocaine use patterns during 1 year, 


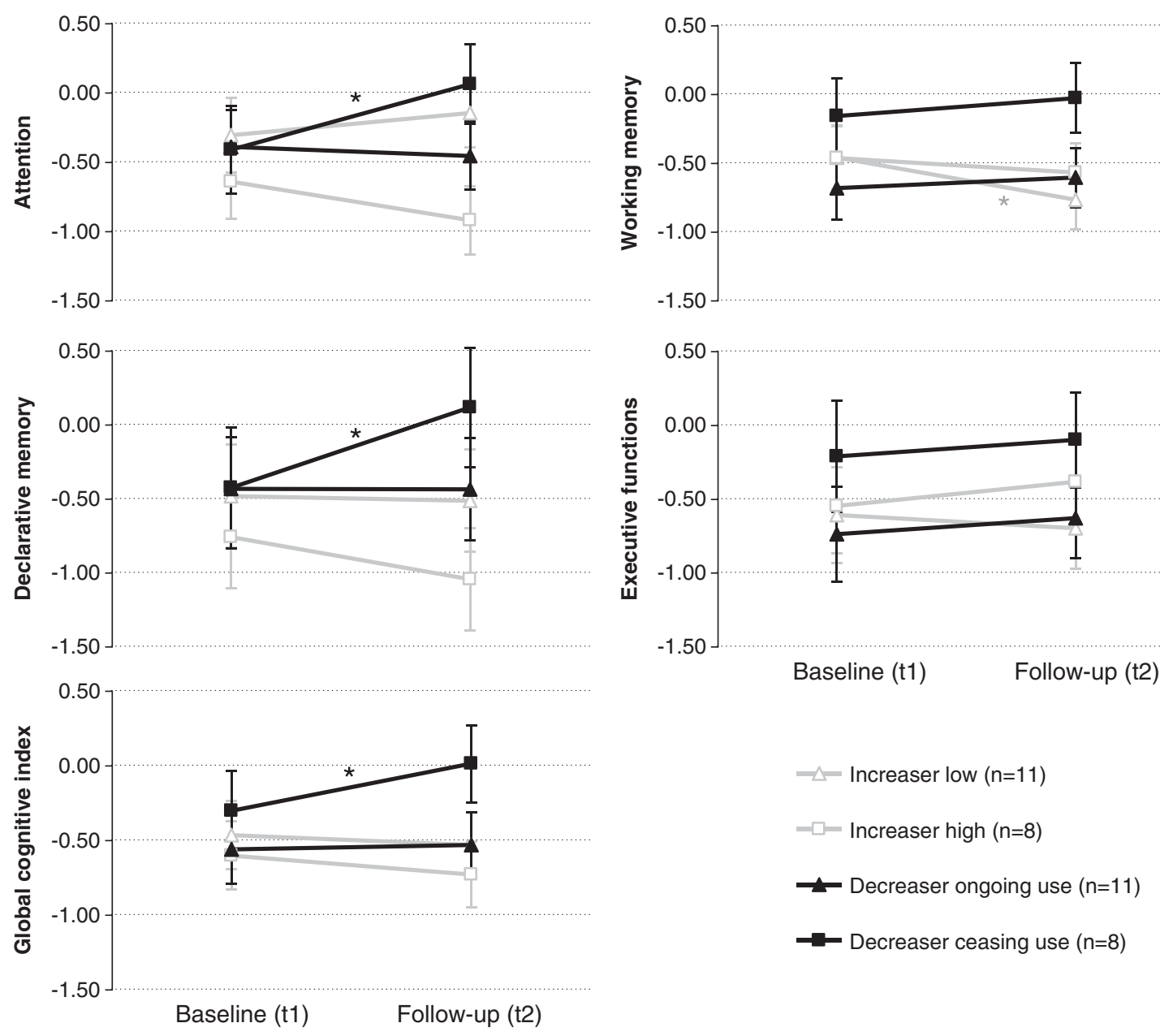

Figure 3 Cognitive functions in low/high cocaine increasers and decreasers with ongoing/ceased cocaine use within I year. Z-scores and SE for the cognitive domains (corrected for ADHD). Z-score transformation was based on control group mean and SD at baseline. Follow-up values were adjusted for the test-retest effect. Change in cognitive test performance from the baseline ( $\mathrm{tl}$ ) to the I-year follow-up (t2): Sidak posthoc tests $(* p<0.05)$. GCl: increaser $_{\text {low }} p=0.61, d=-0.14$, increaser $r_{\text {high }} p=0.41, d=-0.30$, decreaser ongoing $p=0.82, d=0.06$, decreaser ceasing $p<0.05, d=0.93$. Attention: increaser $_{\text {low }} p=0.41, d=0.29$, increaser $r_{\text {high }} p=0.23, d=-0.46$, decreaser ongoing $p=0.74, d=-0.08$, decreaser $_{\text {ceasing }} p<0.05, d=1.10$. Working memory: increaser low $p<0.05, d=-0.60$, increaser high $_{1} p=0.52, d=-0.42$, decreaser ongoing $p=0.58, d=0.20$, decreaser ceasing $p=0.42, d=0.22$. Declarative memory: increaser low $_{p} p=0.90, d=-0.04$, increaser $_{\text {high }} p=0.30, d=-0.29$, decreaser ongoing $_{\text {p }} p=0.99, d=-0.01$, decreaser ceasing $p<0.50$, $d=0.65$. Executive functions: increaser low $p=0.70, d=0.09$, increaser high $p=0.54, d=0.46$, decreaser ongoing $_{\text {o }} p=0.63, d=0.12$, decreaser ceasing $p=0.68$, $d=0.17$.

with the development of cognitive performance. Hair toxicology analyses allowed a precise drug use quantification to detect changes across the test interval and ensured the inclusion of participants with relatively little polytoxic drug use.

This study yielded several major findings: First, increased cocaine use was associated with additional cognitive decline within 1 year, particularly in working memory, supporting the hypothesis that these cognitive impairments were partially cocaine-induced, as recent animal studies have implied (Gould et al, 2012; Liu et al, 2008; Olausson et al, 2007; Porter et al, 2011). This finding is also in line with previous cross-sectional studies showing that the extent, duration, and amount of cocaine use are related to the severity of cognitive dysfunction (Bolla et al, 1999; Colzato et al, 2007; Vonmoos et al, 2013). Second, decreased cocaine use within 1 year was linked to small but consistent cognitive improvements in all four domains confirming the assumption from previous cross-sectional and longitudinal studies that cognitive consequences from crack cocaine use might be partially reversible (De Oliveira et al, 2009; Di Sclafani et al, 2002). Users with moderate lifetime exposure who completely ceased their cocaine consumption seemed to recover entirely and attained similar attention, memory, and global cognitive performance as controls in the follow-up. Because chronic cocaine administration to rhesus monkey produced neuroadaptations in dopamine systems (Letchworth et al, 2001; Nader et al, 2002), the reversibility of cognitive deficits after sustained abstinence suggests that neuroplastic adaptations might be restored if the repeated pharmacological stimulus is discontinued. Third, correlations between the cumulative cocaine dose used during the test interval and cognitive change scores further support the hypothesis that cognitive decline might be drug-induced. Moreover, a substantial correlation between the age of cocaine use onset and change in working 
memory performance in decreasers indicates that early onset might be a risk factor for sustained cognitive impairment after chronic cocaine use.

Users with escalating cocaine use displayed the largest cognitive decrements in working memory, confirming findings from our larger cross-sectional sample (Vonmoos et al, 2013) and from a meta-analysis (Jovanovski et al, 2005) that this domain is strongly affected in dependent cocaine users. The working memory domain was also improved if cocaine consumption was considerably decreased. These data suggest that either working memory is most susceptible to cocaine effects, as it has previously been associated with monoamine functioning (Robbins and Arnsten, 2009), or working memory tasks are the most reliable and sensitive test parameters. In fact, among controls, the test-retest reliability of the declarative memory $(r=0.80)$, GCI $(r=0.78)$, and working memory $(r=0.77)$ was superior compared with executive function $(r=0.59)$ and attention $(r=0.55)$.

Overall, the cognitive changes in our longitudinal study appear to be relatively small. However, at baseline, the increaser group already had a cumulative lifetime cocaine dose of $1.2 \mathrm{~kg}$ - a level at which most cocaine users already display substantial cognitive impairments (Vonmoos et al, 2013). Given that the increasers reported an additional cumulative cocaine dose of $90 \mathrm{~g}$, used between baseline and follow-up, this amount might have been too small to exert additional and measurable cognitive decrements (in conjunction with possible ceiling effects).

The putative reversibility of cognitive impairments in decreasers, particularly in working memory and declarative memory, confirms the results of two previous studies indicating memory improvements in cocaine users at 6months abstinence (De Oliveira et al, 2009; Di Sclafani et al, 2002). However, one study (De Oliveira et al, 2009) had a cross-sectional design, whereas the other (Di Sclafani et al, 2002) postulated improvements but did not correct for testretest effects. Another study (van Gorp et al, 1999) with cocaine users $(n=37)$ found lasting detrimental effects in nonverbal declarative memory but small improvements in a verbal declarative memory test after 45 days of drug abstinence-a finding similar to the RAVLT results in our study. Moreover, a study with cocaine users $(n=30)$ at 1 month of drug abstinence found no significant differences in learning and delayed recall compared with controls (Bolla et al, 1999). Because dependent cocaine users exhibited reduced activity in frontal regions (Volkow et al, 2009) crucial for cognitive functioning (Cabeza and Nyberg, 2000) and given that these reductions persisted at least three to 4 months after detoxification (Volkow et al, 1992), the abstinence duration in the last two studies mentioned here was supposedly too brief to reveal cognitive recovery effects.

The cognitive recovery process seemed to be particularly pronounced in users who ceased taking cocaine; at followup, all of these users had a GCI score within one SD of the control group. However, cocaine users who had been abstinent for at least 6 months also reported a relatively low cumulative lifetime dose of cocaine $(0.7 \mathrm{~kg})$ compared with users with decreased but ongoing cocaine use $(5.9 \mathrm{~kg})$. Because the abstinent user group did not significantly differ from the other cocaine use subgroups in terms of age, sex, verbal IQ, education, and ADHD, their putatively higher cognitive performance might be probably explained by this lower baseline use of cocaine. Nonetheless, it remains unclear if the subjects in this group became abstinent because of their higher overall functioning, or whether there is a 'point of no return' none of these subjects attained (ie, a cumulative cocaine dose beyond which no full recovery can be expected). Nevertheless, we propose that the reversibility of cognitive functions in cocaine users (1) takes some time (at least several months), (2) differs among cognitive domains, (3) depends on the residual level of cocaine use, and (4) is probably related to the amount of lifetime cumulative cocaine dose and age of onset.

This study has some limitations. First, although the group assignment was based on objective hair toxicology covering the last 6 months, for the first 6 months of the time interval we could rely only on self-reports. Second, the importance of hair melanin pigment for the incorporation of cocaine into the hair structure has not been conclusively clarified (Mieczkowski and Newel, 2000). However, because there is no apparent melanin effect regarding cocaine (Mieczkowski and Kruger, 2007), and 30 of 38 cocaine users in the present study had brownish hair, it is unlikely that the group assignment was affected by this potential constraint. We also used a within-subject design, and, thus, inter-individual differences in hair color should play a minor role. Third, our executive function domain comprised only two parameters because we excluded the CANTAB IED from follow-up testing. Future longitudinal studies might therefore employ a more comprehensive neuropsychological test battery focusing on executive functioning. Fourth, although our sample consists of cocaine users with relatively little polytoxic drug use, it should be mentioned that at baseline, cocaine increasers displayed a small but significantly higher use of MDMA (0.04 vs 0.01 tablets per week) and longer use of amphetamine (3.3 vs 1.3 years) than decreasers. Furthermore, at follow-up cocaine increasers revealed a slightly higher use of MDMA and methylphenidate compared with baseline and featured an additional rise in weekly alcohol use. Although the change in MDMA use was less than half a tablet per week, the difference in methylphenidate consumption was explained by a single individual. The rise in weekly alcohol use was based on an increased intake in three of 19 cocaine increasers. However, exclusion of the single methylphenidate user and the alcohol increasing subjects did not change the main results in separate analyses. Thus, although changes in other drugs should be considered as a contributing factor to our results, it seems reasonable that compared with the strong increase in cocaine use, the effect of changed use of other drugs is likely rather small.

In conclusion, our findings suggest that cognitive performance co-varies with changing cocaine use within a 1 -year period. Whereas increased cocaine use was associated with further decrements of cognitive functioning (most pronounced in working memory), decreased cocaine use was linked to improved cognition, particularly in attention and the memory domains. Remarkably, cocaine users who completely ceased their consumption attained the same cognitive performance level as the controls. However, early age of cocaine use onset seem to hamper these recovery processes, at least in the working memory, which is a highly relevant finding for prevention and harm 
reduction interventions. Although previous research has discussed the possibility of neuroenhancement in stimulant users by drugs (Sofuoglu et al, 2013), our findings suggest that drug abstinence might be the best way to recover cognitive performance in stimulant users as abstinence has obviously a more beneficial side-effect profile than any psychopharmacological intervention. Although it has been shown that stimulant treatment can improve cognitive performance in cocaine users at least acutely (Sofuoglu $\mathrm{et} \mathrm{al,}$ 2013), the use of prescription stimulants to treat cognitive deficits in stimulant users might be questioned given that methylphenidate and amphetamines likely produce or even prolong neuroplasticity induced by cocaine or other illegal stimulants as they have similar mechanisms of action (Svetlov et al, 2007). However, the chronic effect or the discontinuation of pro-cognitive stimulant treatment on cognition of cocaine users has not been investigated so far. Finally, the general reversibility of cognitive deficits also indicates that drug-induced neuroadaptations can probably be remodulated by psychotherapeutical or pharmacological interventions, which might help to achieve and maintain abstinence.

\section{FUNDING AND DISCLOSURE}

The study was supported by grants from the Swiss National Science Foundation (SNSF; grant No. PP00P1-123516/1 and PP00P1-146326/1) and the Olga Mayenfisch Foundation. The funders of the study did not influence the design and conduct of the study; the collection, management, analysis, and interpretation of the data; the preparation, review, and approval of the manuscript; and the decision to submit the manuscript for publication. The work of Dr Vonmoos, Dr Hulka, Dr Preller, Ms Minder, and Prof Quednow has been funded by the Swiss National Science Foundation projects as stated above. Matthias Vonmoos and Boris B. Quednow have full access to all of the data in the study and take responsibility for the integrity of the data and the accuracy of the data analysis. The authors declare no conflict of interest.

\section{ACKNOWLEDGEMENTS}

We are grateful to Alex Bücheli (Streetwork Zürich), Rudolf Stohler (Research Group Substance Use Disorders, University Hospital of Psychiatry), Lars Stark and Thilo Beck (ARUD, Zurich), Eric La Serra (Klinik St Pirminsberg, Psychiatrie-Dienste Süd, Kanton St Gallen), Christopher Schütz (Integrated Psychiatry Wintherthur), and Michael Schaub (Swiss Research Institute for Public Health and Addiction, Zürich) for their support of the recruitment of cocaine-using individuals. In addition, we thank Alex Gamma for critical comments to the manuscript and statistical support. Moreover, we thank Daniela Jenni and Kathrin Küpeli for excellent support.

\section{REFERENCES}

American Psychiatric Association (1994). Diagnostic and Statistical Manual of mental disorders: DSM-IV. 4th edn. American Psychiatric Association (APA): Washington, DC.
Bauer LO (1996). Psychomotor and electroencephalographic sequelae of cocaine dependence. NIDA Res Monogr 163: 66-93.

Bolla KI, Funderburk FR, Cadet JL (2000). Differential effects of cocaine and cocaine alcohol on neurocognitive performance. Neurology 54: 2285-2292.

Bolla KI, Rothman R, Cadet JL (1999). Dose-related neurobehavioral effects of chronic cocaine use. J Neuropsychiatry Clin Neurosci 11: 361-369.

Bush DM (2008). The US Mandatory Guidelines for Federal Workplace Drug Testing Programs: current status and future considerations. Forensic Sci Int 174: 111-119.

Cabeza R, Nyberg L (2000). Imaging cognition II: an empirical review of 275 PET and fMRI studies. J Cogn Neurosci 12: 1-47.

Colzato LS, Huizinga M, Hommel B (2009). Recreational cocaine polydrug use impairs cognitive flexibility but not working memory. Psychopharmacology 207: 225-234.

Colzato LS, van den Wildenberg WP, Hommel B (2007). Impaired inhibitory control in recreational cocaine users. PLoS One 2: e1143.

Cooper GA, Kronstrand R, Kintz P (2012). Society of Hair Testing guidelines for drug testing in hair. Forensic Sci Int 218: 20-24.

De Oliveira LG, Barroso LP, Silveira CM, Sanchez ZV, De Carvalho Ponce J, Vaz LJ et al (2009). Neuropsychological assessment of current and past crack cocaine users. Subst Use Misuse 44: 1941-1957.

Degenhardt L, Hall W (2012). Extent of illicit drug use and dependence, and their contribution to the global burden of disease. Lancet 379: 55-70.

Di Sclafani V, Tolou-Shams M, Price LJ, Fein G (2002). Neuropsychological performance of individuals dependent on crack-cocaine, or crack-cocaine and alcohol, at 6 weeks and 6 months of abstinence. Drug Alcohol Depend 66: 161-171.

Faul F, Erdfelder E, Lang AG, Buchner A (2007). G*Power 3: a flexible statistical power analysis program for the social, behavioral, and biomedical sciences. Behav Res Methods 39: 175-191.

Goldstein RZ, Leskovjan AC, Hoff AL, Hitzemann R, Bashan F, Khalsa SS et al (2004). Severity of neuropsychological impairment in cocaine and alcohol addiction: association with metabolism in the prefrontal cortex. Neuropsychologia 42: 1447-1458.

Gould RW, Gage HD, Nader MA (2012). Effects of chronic cocaine self-administration on cognition and cerebral glucose utilization in Rhesus monkeys. Biol Psychiatry 72: 856-863.

Hautzinger M, Bailer M, Worall H, Keller F (1994). BeckDepressions-Inventar (BDI). Bearbeitung der deutschen Ausgabe. Testhandbuch. Beck Depression Inventory. Test manual. 2nd edn. Huber: Bern, Göttingen, Toronto, Seattle.

Heatherton TF, Kozlowski LT, Frecker RC, Fagerstrom KO (1991). The Fagerstrom Test for Nicotine Dependence: a revision of the Fagerstrom Tolerance Questionnaire. Br J Addict 86: 1119-1127.

Helmstaedter C, Lendt M, Lux S (2001). Verbaler Lern- und Merkfähigkeitstest (Verbal learning and memory test). Beltz: Goettingen.

Hoelzle C, Scheufler F, Uhl M, Sachs H, Thieme D (2008). Application of discriminant analysis to differentiate between incorporation of cocaine and its congeners into hair and contamination. Forensic Sci Int 176: 13-18.

Hulka LM, Eisenegger C, Preller KH, Vonmoos M, Jenni D, Bendrick K et al (2014). Altered social and non-social decisionmaking in recreational and dependent cocaine users. Psychol Med 44: 1015-1028.

Hulka LM, Preller KH, Vonmoos M, Broicher SD, Quednow BB (2013). Cocaine users manifest impaired prosodic and crossmodal emotion processing. Front Psychiatry 4: 1-12.

Jovanovski D, Erb S, Zakzanis KK (2005). Neurocognitive deficits in cocaine users: a quantitative review of the evidence. J Clin Exp Neuropsychol 27: 189-204.

Lee RS, Hermens DF, Redoblado-Hodge MA, Naismith SL, Porter MA, Kaur M et al (2013). Neuropsychological and 
socio-occupational functioning in young psychiatric outpatients: a longitudinal investigation. PLoS One 8: e58176.

Lehrl S (1999). Mehrfachwahl-Wortschatz-Intelligenztest. MWT-B. 4th edn. Spitta: Balingen.

Letchworth SR, Nader MA, Smith HR, Friedman DP, Porrino LJ (2001). Progression of changes in dopamine transporter binding site density as a result of cocaine self-administration in rhesus monkeys. J Neurosci 21: 2799-2807.

Liu S, Heitz RP, Sampson AR, Zhang W, Bradberry CW (2008). Evidence of temporal cortical dysfunction in rhesus monkeys following chronic cocaine self-administration. Cereb Cortex 18: 2109-2116.

Mieczkowski T, Kruger M (2007). Interpreting the color effect of melanin on cocaine and benzoylecgonine assays for hair analysis: brown and black samples compared. J Forensic Leg Med 14: 7-15.

Mieczkowski T, Newel R (2000). Statistical examination of hair color as a potential biasing factor in hair analysis. Forensic Sci Int 107: 13-38.

Nader MA, Daunais JB, Moore T, Nader SH, Moore RJ, Smith HR et al (2002). Effects of cocaine self-administration on striatal dopamine systems in rhesus monkeys: initial and chronic exposure. Neuropsychopharmacology 27: 35-46.

Nutt D, King LA, Saulsbury W, Blakemore C (2007). Development of a rational scale to assess the harm of drugs of potential misuse. Lancet 369: 1047-1053.

Olausson P, Jentsch JD, Krueger DD, Tronson NC, Nairn AC, Taylor JR (2007). Orbitofrontal cortex and cognitive-motivational impairments in psychostimulant addiction: evidence from experiments in the non-human primate. Ann N Y Acad Sci 1121: 610-638.

Porter JN, Olsen AS, Gurnsey K, Dugan BP, Jedema HP, Bradberry CW (2011). Chronic cocaine self-administration in rhesus monkeys: impact on associative learning, cognitive control, and working memory. J Neurosci 31: 4926-4934.

Preller K, Hulka L, Vonmoos M, Jenni D, Baumgartner M, Dziobek I et al (2014). Impaired emotional empathy and related social network deficits in cocaine users. Addict Biol 19 (e-pub ahead of print).

Quednow BB, Kuhn KU, Hoenig K, Maier W, Wagner M (2004). Prepulse inhibition and habituation of acoustic startle response in male MDMA ('ecstasy') users, cannabis users, and healthy controls. Neuropsychopharmacology 29: 982-990.

Reske M, Eidt CA, Delis DC, Paulus MP (2010). Nondependent stimulant users of cocaine and prescription amphetamines show verbal learning and memory deficits. Biol Psychiatry 68: 762-769.

Robbins TW, Arnsten AF (2009). The neuropsychopharmacology of fronto-executive function: monoaminergic modulation. Annu Rev Neurosci 32: 267-287.
Roesler M, Retz W, Retz-Junginger P, Thome J, Supprian T, Nissen $\mathrm{T}$ et al (2004). Instrumente zur Diagnostik der Aufmerksamkeitsdefizit-/Hyperaktivitätsstörung (ADHS) im Erwachsenenalter [Tools for the diagnosis of attention-deficit/hyperactivity disorder in adults. Self-rating behaviour questionnaire and diagnostic checklist]. Nervenarzt 75: 888-895.

Soar K, Mason C, Potton A, Dawkins L (2012). Neuropsychological effects associated with recreational cocaine use. Psychopharmacology 222: 633-643.

Sofuoglu M, DeVito EE, Waters AJ, Carroll KM (2013). Cognitive enhancement as a treatment for drug addictions. Neuropharmacology 64: 452-463.

Substance Abuse and Mental Health Services Administration (2008). Mandatory Guidelines for Federal Workplace Drug Testing Programs. Federal Register 73: 71858-71907.

Sussner BD, Smelson DA, Rodrigues S, Kline A, Losonczy M, Ziedonis D (2006). The validity and reliability of a brief measure of cocaine craving. Drug Alcohol Depend 83: 233-237.

Svetlov SI, Kobeissy FH, Gold MS (2007). Performance enhancing, non-prescription use of Ritalin: a comparison with amphetamines and cocaine. J Addict Dis 26: 1-6.

Teichner G, Horner MD, Roitzsch JC, Herron J, Thevos A (2002). Substance abuse treatment outcomes for cognitively impaired and intact outpatients. Addict Behav 27: 751-763.

United Nations Office on Drugs and Crime (2013). World Drug Report 2013. United Nations Publication: Vienna.

van Gorp WG, Wilkins JN, Hinkin CH, Moore LH, Hull J, Horner MD et al (1999). Declarative and procedural memory functioning in abstinent cocaine abusers. Arch Gen Psychiatry 56: 85-89.

Volkow ND, Fowler JS, Wang GJ, Baler R, Telang F (2009). Imaging dopamine's role in drug abuse and addiction. Neuropharmacology 56: 3-8.

Volkow ND, Hitzemann R, Wang GJ, Fowler JS, Wolf AP, Dewey SL et al (1992). Long-term frontal brain metabolic changes in cocaine abusers. Synapse 11: 184-190.

Vonmoos M, Hulka LM, Preller KH, Jenni D, Baumgartner MR, Stohler R et al (2013). Cognitive dysfunctions in recreational and dependent cocaine users: role of attention-deficit hyperactivity disorder, craving and early age at onset. $\mathrm{Br} J$ Psychiatry 203: 35-43.

Wechsler DA (1997). Wechsler Memory Scale. Technical Manual. 3rd edn. Psychological Cooperation: San Antonio, TX, USA.

Wilson JJ (2007). ADHD and substance use disorders: developmental aspects and the impact of stimulant treatment. Am J Addict 16(Suppl 1): 5-11.

Woicik PA, Moeller SJ, Alia-Klein N, Maloney T, Lukasik TM, Yeliosof O et al (2009). The neuropsychology of cocaine addiction: recent cocaine use masks impairment. Neuropsychopharmacology 34: 1112-1122.

Supplementary Information accompanies the paper on the Neuropsychopharmacology website (http://www.nature.com/npp) 